\title{
A Radiological Study of Prevelance of Sinusitis in Children
}

\author{
Usman Nema*,Ajmal Mohd* \\ *Department Of Anatomy, JNMC, AMU, Aligarh, UP, India \\ *Corresponding author: Usman Nema*
}

\begin{abstract}
Introduction: Respiratory tract infections are very common in children in India. However, in the setting of prolonged symptoms suggesting a bacterial infection, radiographic images can lend credence to the diagnosis. Very few reports of the prevalence of sinusitis in children with recurrent respiratory tract symptoms (RRTS) have been reported in India, and, hence, this study was carried out.

Methodology: This descriptive study was done at JNMC AMU, a tertiary-care hospital Aligarh,U.P. over a period of 1 year. All children in the age group of 3-15 years who showed RRTS (>3 episodes in 6 months or 6 episodes in 1 year) were included in the study. A total number of 60 children were included in the study. Radiological diagnosis of sinusitis by X-ray PNS and CT-scan was done to appreciate the presence of complete opacification, mucosal thickening (at least $4 \mathrm{~mm}$ ), and air fluid levels .

Results and conclusion: Out of 60 children with RRTS 36 cases (60\%) were diagnosed as cases of sinusitis radiologically, 24 cases were not diagnosed radiologically.
\end{abstract}

Keywords: sinusitis , X-ray PNS

\section{Introduction}

The paranasal sinuses are air-filled spaces located within the bones of the skull and face. They are centered on the nasal cavity and have various functions, including lightening the weight of the head, humidifying and heating inhaled air, increasing the resonance of speech, and serving as a crumple zone to protect vital structures in the event of facial trauma. ${ }^{[1,2]}$

The paranasal sinuses are joined to the nasal cavity via small orifices called ostia. These become blocked easily by allergic inflammation, or by swelling in the nasal lining that occurs with a cold. If this happens, normal drainage of mucus within the sinuses is disrupted, and sinusitis may occur. Because the maxillary posterior teeth are close to the maxillary sinus, this can also cause clinical problems if any disease processes are present, such as an infection in any of these teeth. These clinical problems can include secondary sinusitis, the inflammation of the sinuses from another source such as an infection of the adjacent teeth. ${ }^{[3]}$

The health of children can affect badly due to pediatric sinusitis. Although sinusitis has been found to exist for more than last two decades, there are still arguments that persist in relation to its pathogenesis, presentation, diagnosis, and treatment. An accurate diagnosis of sinusitis would help the clinician to find children who really benefit from the treatment and prevent morbidity from recurrent respiratory tract symptoms (RRTS).

Recurrent respiratory infections affect the daily activities of children. So it is important to see that they may not be suffering from sinusitis. Diagnosis of sinusitis and subsequent care can be very helpful.

Recurrent upper respiratory tract symptoms are defined as symptoms such as cough, cold, and sneezing in a child presenting for more than 3-4 episodes in 6 months or 8-10 episodes in a year, with the interval period being normal (symptom free). RRTS may be owing to allergy, , asthma, inflammation, or infection (viral/bacterial ).

Respiratory tract infections are very common in children in India.. An average child will have 6-8 upper respiratory infections per year $(1,2)$ and it is reported that these infections get complicated by acute bacterial sinusitis. . Bozdoğan et al.,[3] in their study to evaluate the main causes underlying RRTS during childhood, concluded that asthma and allergic diseases were the most common causes (45\%) of recurrent complaints of respiratory tract. The abnormal radiographic images reflect inflammation and do not discern between viral,[4] bacterial,[5] allergic,[6] or other causes. However, in the setting of prolonged symptoms suggesting a bacterial infection, radiographic images can lend credence to the diagnosis. Very few reports of the prevalence of sinusitis in children with recurrent respiratory tract symptoms (RRTS) have been reported in India, and, hence, this study was carried out.

In present study we will try to evaluate the prevalence of sinusitis in children aged 3 to 15 years by clinical and radiological( paranasal sinuses (X-ray PNS) and CT SCAN) methods in children with RRTS, at JNMC AMU ALIGARH. 


\section{Materials And Methods}

This descriptive study was done at JNMC AMU, a tertiary-care hospital Aligarh,U.P. over a period of 1 year. All children in the age group of 3-15 years who showed RRTS ( $>3$ episodes in 6 months or 6 episodes in 1 year) were included in the study. A total number of 60 children were included in the study. Consent of patients and their guardians was taken to utilize their clinical and radiological findings for this study.

Radiological diagnosis of sinusitis by X-ray PNS and CT- scan was done to appreciate the presence of complete opacification, mucosal thickening (at least $4 \mathrm{~mm}$ ), and air fluid levels.

\section{Results}

Out of 60 children with RRTS 36 cases (60\%) were diagnosed as cases of sinusitis radiologically , 24 cases were not diagnosed radiologically .

\begin{tabular}{|l|l|l|l|}
\hline Age Group & Number Of Patients With Rrts & $\begin{array}{l}\text { Number Ofpatients } \\
\text { Radiologically Diagnosed As } \\
\text { Sinusitis }\end{array}$ & $\%$ \\
\hline 3-6 Years & 31 & 16 & 51.6 \\
\hline 7-15 Years & 29 & 20 & 68.9 \\
\hline Total Patients & 60 & 36 & 60 \\
\hline
\end{tabular}

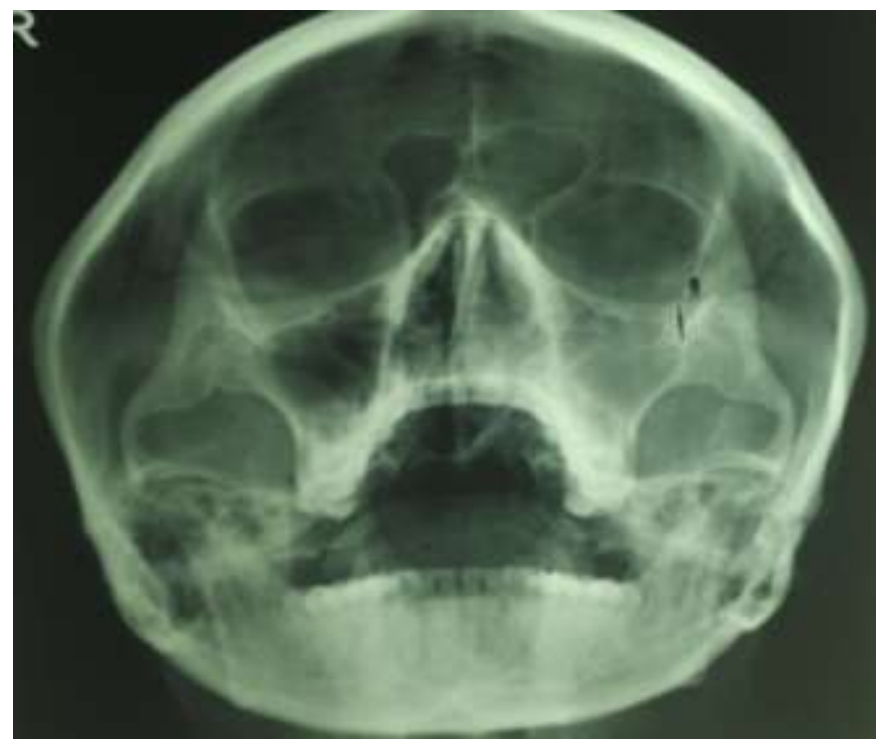

Figure 1: X-Ray PNS showing left side opacifications in maxillary sinus

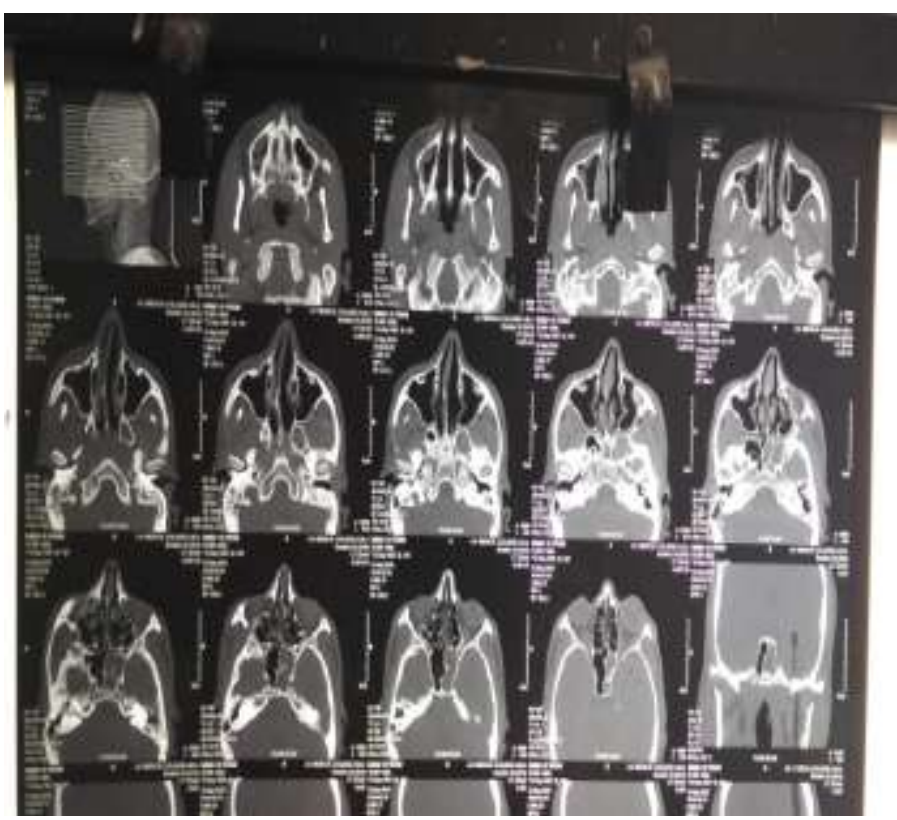

Figure 2: Ct Scan Pns 


\section{Discussion}

In our study, X-ray diagnosis of sinusitis was present in $60 \%$ of children who were clinically suspected to show sinusitis, which is similar to the study done by Arruda et al.[5] Positive significant correlation between the symptoms of cough and purulent discharge with an opaque sinus on radiography in 27 children with clinically suspected sinusitis were found, which is similar to the study done by Conrad and Jenson. [8] Of the 57 recruited children with RRTS, X-ray abnormality was shown in $42(73.68 \%)$ cases. In children with RRTS less than 6 years of age 51.6 show radiological features whereas in 1-15 years old radiologically active sinusitis is appreciated in $68.9 \%$ cases. We can say that incidence of sinusitis increases with age . It may be attributed to more exposure to atmospheric pollution. There is an association between clinical and X-ray diagnoses of sinusitis. Hence, clinical diagnosis of sinusitis is as good as X-ray diagnosis of sinusitis in patients with RRTS.

\section{Conclusion}

The positive predictive value of clinical diagnosis of sinusitis by accepted criteria is $60 \%$, when compared with radiological diagnosis of sinusitis in children with RRTS. Therefore, we conclude that it obviates the need of diagnosing sinusitis radiologically in children with RRTS and is worth treating the children with above-mentioned symptoms based on clinically diagnosed sinusitis by accepted criteria .

\section{Limitations of the Study}

Limitations are that this is a descriptive study with limited number of study population conducted in a limited period, and the study was not blinded. In future this study can be extended to larger population. Moreover we can further appreciate the types of sinusitis in children.

\section{Acknowledgements}

1. Professor Mohammad Aslam, Professor Department of ENT, JNMC, AMU for giving permission to conduct present study in his OPD and for providing necessary X-Rays and CT-scans for our study.

2. Professor Nafis A Faruqi Professor and Head of Department Anatomy JNMC AMU for allowing us to use pieces of his great intellect and knowledge . Conceptual knowledge regarding clinical anatomy of nose and paranasal sinuses provided by Professor Nafis A Faruqi during our academic life will always remain a stepping stone whenever we venture into researches regarding paranasal sinuses.

3. Dr Farhan Kirmani Associate Professor for his valuable suggestions

4. Last but not the least we want to thank all the paediatric patients and their guardians who had allowed us to use their clinical and radiological findings in our study.

\section{References}

[1]. Dalgorf DM, Harvey RJ. Chapter 1: Sinonasal anatomy and function. Am J Rhinol Allergy. 2013 May-Jun. 27 Suppl 1:S3-6. [Medline].

[2]. Reddy UD, Dev B. Pictorial essay: Anatomical variations of paranasal sinuses on multidetector computed tomography-How does it help FESS surgeons?. Indian J Radiol Imaging. 2012 Oct. 22(4):317-24. [Medline]. [Full Text].

[3]. Illustrated Anatomy of the Head and Neck, Fehrenbach and Herring, Elsevier, 2012, p. 68

[4]. Couriel JM. Lower respiratory tract infections in childhood. In: Infections of the Respiratory Tract, Ellis M (Ed.). Cambridge: Cambridge University Press, 1998. pp. 406-27.

[5]. Woroniecka M, Ballow M. Office evaluation of children with recurrent infection. Pediatr Clin North Am 2000;47(6):1211-24.

[6]. Bozdoğan G, Reisli I, Doğu F, lkincioğullari A, Babacan E. Evaluation of the children with recurrent respiratory tract infection. J Med Sci 2003;3(5-6):411-7.

[7]. Gwaltney JM, Phillips CD, Miller RD, Riker DK. Computed tomographic study of the common cold. N Engl J Med 1994;330(1):25-30.

[8]. Arruda LK, Mimica IM, Sole D, Weckx LL, Schoettler J, Heiner DC, et al. Abnormal maxillary sinus radiographs in children: do they represent bacterial infection? Pediatrics 1990;85(4):553-8.

[9]. Havas TE, Motbey JA, Gullane PJ. Prevalence of incidental abnormalities on computed tomographic scans of the paranasal sinuses. Arch Otolaryngol Head Neck Surg 1988;114(8):856-9.

[10]. Lanza DC, Kennedy DW. Adult rhinosinusitis defined. Otolaryngol Head Neck Surg 1997;117(3 Pt 2):S51-7.

[11]. Conrad DA, Jenson HB. Management of acute bacterial rhinosinusitis. Curr Opin Pediatr 2002;14(1):86-90. 